\title{
Dementia Care by Healthy Elderly Caregivers Is Associated with Improvement of Patients' Memory and the Caregivers' Quality of Life: A Before and After Study
}

\author{
Seung-Gul Kang ${ }^{1}$, Kyoung-Sae $\mathrm{Na}^{1}$, Jae Myeong Kang ${ }^{1}$, \\ Byeong Kil Yeon ${ }^{1}$, Jun-Young Lee ${ }^{2}$, and Seong-Jin Cho ${ }^{1}$ \\ ${ }^{1}$ Department of Psychiatry, Gil Medical Center, Gachon University School of Medicine, Incheon, Republic of Korea \\ ${ }^{2}$ Department of Psychiatry, Seoul National University College of Medicine and SMG-SNU Boramae Medical Center, Seoul, Republic of Korea
}

\begin{abstract}
Objective The provision of care for elderly people with dementia by healthy elderly caregivers is one of the new health-care paradigms in South Korea. The aim of this study was to determine whether this type of care, which includes cognitive stimulation, would improve the cognitive function of dementia patients and the quality of life of the healthy elderly caregiver.

Methods Totals of 132 dementia patients and 197 healthy elderly caregivers participated in this study. We evaluated the cognitive function of the dementia patients at baseline and after providing the program for 6 months using the Korean version of the Consortium to Establish a Registry for Alzheimer's disease, 1st Edition (CERAD-K). We also evaluated the quality of life of the healthy elderly caregivers using the World Health Organization Quality of Life-Short Version (WHOQOL-BREF) at baseline and after 6 months.

Results The word-list memory results of CERAD-K for the included dementia patients improved after 6 months $(Z=-2.855, p=0.004)$. The WHOQOL-BREF score among the elderly caregiver also improved significantly $(Z=-2.354, p=0.019)$.

Conclusion These data suggest that dementia care is associated with improvements in both the cognitive function of dementia patients and the quality of life of the healthy elderly caregivers.

Psychiatry Investig 2017;14(4):458-462
\end{abstract}

Key Words Care, Dementia, Elderly, Memory, Quality of life.

\section{INTRODUCTION}

Dementia is an acquired brain disease characterized by the progressive deterioration of cognitive function and functional impairment. Dementia patients need nursing care due to the severity of their behavioral and psychological symptoms increasing as the illness progresses. The prevalence of dementia is increasing more rapidly in Korea than in many other countries due to the rapid increases in life expectancy and the proportion of the aging population. ${ }^{1}$ This has resulted in dementia becoming a serious social problem in Korea.

Korean society is experiencing difficulty in caring for the

Received: March 12, 2016 Revised: May 10, 2016

Accepted: June 28, 2016 Available online: May 16, 2017

$\triangle$ Correspondence: Seong-Jin Cho, MD, PhD

Department of Psychiatry, Gil Medical Center, Gachon University School of Medicine, 21 Namdong-daero 774beon-gil, Namdong-gu, Incheon 21565, Republic of Korea

Tel: +82-32-460-8420, Fax: +82-32-472-3396, E-mail: sjcho@gilhospital.com

(a) This is an Open Access article distributed under the terms of the Creative Commons Attribution Non-Commercial License (http://creativecommons.org/licenses/by$\mathrm{nc} / 4.0$ ) which permits unrestricted non-commercial use, distribution, and reproduction in any medium, provided the original work is properly cited. elderly with dementia due to the increasing number of nuclear and dual-career families. This new situation means that the elderly with dementia are often neglected, while the socioeconomic burden due to the increasing prevalence of dementia has increased. The Korean government has increased the nursing and social support to dementia patients. One of these governmental supports involves healthy elderly caring for those with dementia, and this is considered a promising and efficient program for dementia care, since many retired healthy people are enthusiastic about being employed in caring for the elderly with dementia. ${ }^{2}$ Elderly caregivers inherently have a good understanding about elderly people with dementia, and they are also interested in dementia treatments because they realize that they could be in the same situation as dementia patients in the future. An elderly caregiver also feels a sense of accomplishment in working as a caregiver. In addition to these benefits, the government can reduce the budget for dementia care since employing elderly caregivers costs less than employing younger caregivers.

The programs for dementia care by the healthy elderly could 
have various compositions. Previous such programs in Korea have included helping in the activities of daily living, providing physical activity and mental stimulation, and being a conversation companion. A cognitive intervention that includes cognitive stimulation has recently been accepted as complementary medical treatment for dementia, since this is a progressing neurodegenerative disorder and its response to pharmacological treatment is quite limited. ${ }^{3,4}$ While a program involving dementia care by the elderly that includes simple cognitive stimulation is expected to have beneficial effects on cognitive function and the quality of life of the care recipients, this has not been confirmed by scientific evidence.

The first aim of this study was to determine the relationship between dementia care by the elderly that includes cognitive stimulation and the cognitive function and quality of life of patients with mild-to-moderate dementia. The second aim of this study was to determine the relationship between this care program and the quality of life of the elderly caregivers.

\section{METHODS}

\section{Subjects}

All of the dementia patients were enrolled from the 10 dementia counseling centers in Incheon, South Korea. In total, 301 elderly patients who were older than 60 years and had dementia as diagnosed by physicians in accordance with 10th revision of the International Statistical Classification of Diseases and Related Health Problems criteria were evaluated for the eligibility in our study. ${ }^{5}$ All of them had been taking choline esterase inhibitors and/or NMDA antagonists. In the screening interview, subjects with any of the following characteristics were excluded: admission to long-term care facilities, symptoms that were too severe to allow neuropsychological testing, and not providing written informed consent to participate in the study (both themselves and their family members). Finally, 132 elderly people with dementia were enrolled in the study. Healthy elderly caregivers older than 60 years were recruited from the Incheon Senior Resources Development Center and evaluated for medical illness by historytaking and the elderly who had the serious medical disease were excluded. Finally, 197 healthy elderly who were willing to participate in dementia care and the research program provided written informed consents to participate. The study protocol was approved by the Institutional Review Board of Gil Medical Center.

\section{Care program}

The main aim of this care program was to improve the cognitive function, quality of life, emotional state, and activities of daily living of dementia patients. A secondary aim was to improve the quality of life and self-esteem of the caregivers. The applied program included cognitive stimulation and general care.

The cognitive stimulation comprised reading a book or newspaper to patients or having them read the same, providing useful information, and participating in origami, painting, naming, communication practice, and a life review. The contents of the cognitive stimulation were selected based on previous cognitive stimulation studies. ${ }^{3,6}$ The general care comprised assistance in taking pills, maintaining personal hygiene, providing emotional support, supporting social activities, performing physical activities (e.g., strolling and other forms of exercise), and singing.

An educational course taking 36 hours was provided to the caregivers, and they were supplied with a manual containing a comprehensive description of the care procedures. The total duration, frequency, and session duration of the program were 6 months, three times per week, and 4 hours per session, respectively. A health-care practitioner gave regular call once per month to ensure that all of the healthy elderly caretakers were providing the program correctly.

\section{Evaluation: cognitive and quality of life assessment}

The included dementia patients and caregivers were evaluated at baseline and 6 months after implementing the program. In order to evaluate changes in the cognitive function of subjects, the Korean version of the Consortium to Establish a Registry for Alzheimer's disease Assessment Packet, 1st Edition (CERAD-K) and the Montreal Cognitive Assessment for Dementia were applied to all of the dementia patients by clinical psychologists. ${ }^{7.8}$ These clinical psychologists were well trained in neuropsychological tests by certified clinical psychologists, and they had sufficient experience in applying these tests to dementia patients. The Functional Activities Questionnaire (FAQ), ${ }^{9}$ Disability Assessment for Dementia $(\mathrm{DAD}-\mathrm{K}){ }_{,}^{10}$ and World Health Organization Quality of LifeShort Version (WHOQOL-BREF) ${ }^{11}$ were used to evaluate cognitive function, activities of daily living, and quality of life in the dementia patients. The quality of life of the caregivers was evaluated using the WHOQOL-BREF.

\section{Statistical analysis}

Sociodemographic data were analyzed using the chi-square test for dichotomous variables and the independent $t$ test for continuous variables. Since the Shapiro-Wilk test indicated that most of the scores for the neurocognitive tests, social functioning, and quality of life were not normally distributed, the nonparametric Wilcoxon's signed-rank test was used to compare these variables between before and after the intervention. The cutoff for statistical significance was set as $p<0.05$ 
(two tailed). All statistical analyses were performed using the Statistical Package for the Social Sciences (SPSS) for Windows (SPSS, Chicago, IL, USA).

\section{RESULTS}

\section{Demographic data}

The sociodemographic data of the dementia patients and caregivers are listed in Table 1. There were 113 (85.6\%) female and 19 (14.4\%) male dementia patients, and they were aged $79.9 \pm 7.1$ (mean \pm SD) years. Their duration of education was $7.2 \pm 3.6$ years, and $62(47.0 \%)$ of them had received no education. Sixty-one dementia patients were evaluated after participating in the care program for 6 months; this excluded those who had withdrawn their consent and who could not be evaluated for various other reasons including a lack of cooperation.

There were 169 (85.8\%) female and 28 (14.2\%) male caregivers, and they were aged $68.6 \pm 4.8$ years. Their duration of education was $8.0 \pm 3.7$ years. One hundred and six caregivers were evaluated after participating in the care program for 6 months; this excluded those who declined to be evaluated for several reasons, including withdrawal of consent.

\section{Results for dementia patients}

\section{Neuropsychological tests}

CERAD-K was used to evaluate changes in the cognitive function of the dementia patients after the care program. The results obtained when applying the neurocognitive function

Table 1. Sociodemographic data of the participants

\begin{tabular}{lcc}
\hline & $\begin{array}{c}\text { Patients } \\
(\mathrm{N}=132)\end{array}$ & $\begin{array}{c}\text { Caregivers } \\
(\mathrm{N}=197)\end{array}$ \\
\hline Age, years & $79.9 \pm 7.1$ & $68.6 \pm 4.8$ \\
Gender, female & $113(85.6)$ & $169(85.8)$ \\
Education, years & $7.2 \pm 3.6$ & $8.0 \pm 3.7$ \\
Marital status & & \\
$\quad$ Married & $33(25.0)$ & $130(66.0)$ \\
Widow (er) & $82(62.1)$ & $52(26.4)$ \\
Separated/divorced & $2(1.52)$ & $10(5.1)$ \\
Not married & $1(0.8)$ & $2(1.0)$ \\
$\quad$ Missing data & $14(10.6)$ & $3(1.5)$ \\
Cohabitation & & \\
Living alone & $48(36.4)$ & $37(18.8)$ \\
With spouse & $26(19.7)$ & $100(50.8)$ \\
With spouse and others & $10(7.6)$ & $31(15.7)$ \\
With others & $34(25.8)$ & $27(13.7)$ \\
Missing data & $14(10.6)$ & $2(1.0)$ \\
\hline All &
\end{tabular}

All data are mean \pm SD or number (\%) values tests [verbal fluency, Boston Naming Test, Korean version of the Mini-Mental State Examination (MMSE-KC) in the CERAD$\mathrm{K},{ }^{12}$ word-list memory, constructional praxis, word-list recall, word-list recognition, and constructional recall test] to patients with dementia before and after the program are listed in Table 2. Application of the nonparametric Wilcoxon's signedrank test revealed that the word-list memory results of CERAD$\mathrm{K}$ for dementia patients improved after 6 months $(\mathrm{Z}=-2.855$, $\mathrm{p}=0.004$ ), whereas there were no significant changes in any of the other neurocognitive function tests (Table 2).

\section{Function, disability, and quality of life}

The FAQ ( $\mathrm{F}=-0.259, \mathrm{p}=0.795)$, DAD-K $(\mathrm{F}=-1.730, \mathrm{p}=$ 0.084 ), and WHOQOL-BREF ( $\mathrm{F}=-0.777, \mathrm{p}=0.437)$ scores of dementia patients did not show any statistically significant changes after the program (Table 2).

\section{Results for the caregivers}

The WHOQOL-BREF score was significantly higher for the caregivers $(70.57 \pm 17.61)$ than for the dementia patients $(52.88 \pm$ 7.63, $\mathrm{p}<0.001)$ at baseline, and it improved for the caregivers after 6 months $(Z=-2.354, p=0.019)$ when applying the nonparametric Wilcoxon's signed-rank test, especially in the environmental domain $(\mathrm{Z}=-3.865, \mathrm{p}=0.0001)$ (Table 3$)$.

\section{DISCUSSION}

This study found that the provision of dementia care by healthy elderly that includes cognitive stimulation improved the word-list memory of dementia patients and also the quality of life of the caregivers themselves. This is the first study to investigate the effects of dementia care provided by healthy elderly.

Previous studies of the effects of cognitive interventions (cognitive stimulation, cognitive training, and cognitive rehabilitation) on dementia patients produced inconsistent results. Moreover, the results of meta-analyses have varied depending on which studies were included. One meta-analysis of 15 randomized controlled trials (RCTs) that included 718 subjects found that cognitive stimulation showed some benefit in improving cognitive function and well-being. ${ }^{13}$ In contrast, a meta-analysis of 11 RCTs found that cognitive training was not associated with either positive or negative effects. ${ }^{14}$ This inconsistency is due to differences in the applied methods, the duration of cognitive stimulation, the dementia severity of the participants, and the neurocognitive function tests used for the evaluations in the meta-analysis. Moreover, many studies have adopted brief evaluation methods for global assessments of cognition and dementia severity (e.g., MMSE and Clinical Dementia Rating ${ }^{15}$ ), rather than more accurate 
Table 2. Comparison of neurocognitive function in patients with dementia between before and after dementia care provided by healthy elderly caregivers that included cognitive stimulation

\begin{tabular}{|c|c|c|c|c|}
\hline & Baseline & 6 months & $\mathrm{Z}$ & $\mathrm{p}$ \\
\hline \multicolumn{5}{|l|}{ Cognitive function } \\
\hline \multicolumn{5}{|l|}{ CERAD-K } \\
\hline Verbal fluency & $5.92 \pm 2.85$ & $6.74 \pm 5.35$ & -0.646 & 0.518 \\
\hline Boston Naming Test & $5.82 \pm 2.90$ & $6.18 \pm 3.17$ & -1.168 & 0.243 \\
\hline MMSE-KC & $14.62 \pm 5.10$ & $13.93 \pm 4.22$ & -1.611 & 0.107 \\
\hline Word-list memory & $7.50 \pm 4.30$ & $12.45 \pm 8.43$ & -2.855 & 0.004 \\
\hline Constructional praxis & $6.81 \pm 2.62$ & $6.89 \pm 2.57$ & -0.097 & 0.923 \\
\hline Word-list recall & $3.85 \pm 1.91$ & $4.08 \pm 2.25$ & -0.900 & 0.368 \\
\hline Word-list recognition & $5.74 \pm 2.98$ & $7.00 \pm 2.72$ & -1.818 & 0.069 \\
\hline Constructional recall & $4.11 \pm 1.27$ & $3.78 \pm 1.56$ & -0.736 & 0.461 \\
\hline MoCA & $7.16 \pm 5.57$ & $6.37 \pm 4.48$ & -1.484 & 0.138 \\
\hline \multicolumn{5}{|l|}{ Social function } \\
\hline FAQ & $18.89 \pm 8.39$ & $19.27 \pm 8.79$ & -0.259 & 0.795 \\
\hline $\mathrm{DAD}$ & $25.67 \pm 10.40$ & $23.81 \pm 12.34$ & -1.730 & 0.084 \\
\hline \multicolumn{5}{|l|}{ Quality of life } \\
\hline \multicolumn{5}{|l|}{ WHOQOL-BREF } \\
\hline Physical health & $17.52 \pm 2.62$ & $17.93 \pm 2.20$ & -0.636 & 0.525 \\
\hline Psychological health & $15.46 \pm 3.96$ & $15.30 \pm 2.45$ & -0.218 & 0.827 \\
\hline Social relationships & $6.11 \pm 1.62$ & $6.55 \pm 1.72$ & -1.458 & 0.145 \\
\hline Environment & $15.61 \pm 3.70$ & $15.05 \pm 3.26$ & -1.016 & 0.310 \\
\hline Total & $53.80 \pm 7.36$ & $54.60 \pm 6.91$ & -0.777 & 0.437 \\
\hline
\end{tabular}

All data are mean $\pm S D$ values. CERAD-K: Korean version of the Consortium to Establish a Registry for Alzheimer's disease, MMSE-KC: Korean version of the Mini-Mental Status Examination in the CERAD-K, MoCA: Montreal Cognitive Assessment, FAQ: Functional Activity Questionnaire, DAD: Disability Assessment for Dementia, WHOQOL-BREF: World Health Organization Quality of Life-Short Version

Table 3. Comparison of quality of life in caregivers before and after they had provided dementia care

\begin{tabular}{lcccc}
\hline \multicolumn{1}{c}{ Quality of life } & Baseline & 6 months & $\mathrm{Z}$ & $\mathrm{p}$ \\
\hline WHOQOL-BREF & & & & -1.545 \\
Physical health & $24.91 \pm 3.45$ & $25.58 \pm 4.42$ & -1.558 & 0.122 \\
Psychological health & $20.75 \pm 3.39$ & $21.48 \pm 3.31$ & -0.017 & 0.119 \\
Social relationships & $10.16 \pm 1.53$ & $10.19 \pm 1.96$ & -3.865 & 0.986 \\
Environment & $25.19 \pm 3.99$ & $26.98 \pm 4.11$ & -2.354 & 0.0001 \\
Total & $80.94 \pm 10.62$ & $83.99 \pm 12.08$ & 0.019 \\
\hline
\end{tabular}

All data are mean \pm SD values. WHOQOL-BREF: World Health Organization Quality of Life-Short Version

and objective neuropsychological batteries. ${ }^{16}$

The present study found that word-list memory was the only cognitive domain that improved significantly after applying the program that included cognitive stimulation. This might be attributable to the characteristics of the cognitive stimulation protocol used, which was focused on language domains such as reading, naming, and communication practice. A previous meta-analysis found that immediate verbal memory scores improved significantly after cognitive stimulation. ${ }^{14}$

The well-being and the quality of life of the caregivers and dementia patients after applying cognitive stimulation have varied in previous studies. ${ }^{17}$ Since the caregivers of dementia patients experience high levels of stress and burden, some of them might experience slight deterioration in their own wellbeing. ${ }^{18}$ However, Milders et al. ${ }^{17}$ found no meaningful changes after cognitive stimulation, while Quayhagen et al. ${ }^{19}$ found improved well-being among caregivers. In our study the quality of life of the caregivers improved significantly, especially in the environmental domain. This might be attributable to the characteristics of the included caregivers: many of the caregivers in previous studies were family members, whereas in 
this study they were healthy elderly volunteers who were willing to care for dementia patients and were enthusiastic about participating in paid employment after they had retired. Although elderly caregivers had experienced the minor stress from the caregiving and work, they are thought to have felt relatively more sense of achievement from providing care for and seeing improvements in patients suffering from dementia, which the elderly are highly interested in.

One strength of our study is that it is the first investigation of the effects of the new dementia care model that recently has become a popular and promising care method in the aging society of Korea. In addition, this study adopted an objective and accurate neuropsychological test (i.e., CERAD-K), which is more reliable than global cognition scales such as MMSE. The duration of the intervention was 6 months, which is longer than in previous studies, and this is considered to be long enough for evaluating cognitive improvements in neurodegenerative diseases of the brain such as dementia.

The data reported herein suggest that dementia care provided by healthy elderly caregivers that includes simple cognitive stimulation produces improvements in both the cognitive function of dementia patients and the quality of life of the caregivers themselves. These data indicate that the provision of dementia care by elderly caregivers who are motivated and interested in this care is helpful to both the dementia patients and the caregivers. This evidence is particularly meaningful given that aging societies will require greater use of this type of care model in the future.

The care program implemented by healthy elderly in this study only affected the neuropsychological domain of wordlist memory. Previous studies of the effects of cognitive stimulation on dementia patients have produced inconsistent findings. Therefore, the future development of dementia care programs that are provided by the elderly will need diverse trials and research into how best to incorporate cognitive stimulation so as to enhance the cognitive function of dementia patients. The future researches on this topic are recommended to be performed as the controlled case-control study since our study could not exclude the medication effect on the cognitive function, which is the limitation of our study. In addition, a care program that could improve the quality of life and the satisfaction of dementia patients should be another focus for this type of care model.

\section{Acknowledgments}

This research was supported by the Ministry of the Health and Welfare. This work was supported by the Gachon University Gil Medical Center
(Grant number: 2013-43).

\section{REFERENCES}

1. Korea National Statistical Office. Statistics for the Elderly 2012. Daejeon: Statistics Korea; 2012.

2. Chae HS, Yoon SD, Kang JH. Evaluation of the elderly care program for seniors in rural areas. Kor J Comm Liv Sci 2008;19:223-234.

3. Kawashima R. Mental exercises for cognitive function: clinical evidence. J Prev Med Public Health 2013;46(Suppl 1):S22-S27.

4. Kurz A, Thone-Otto A, Cramer B, Egert S, Frolich L, Gertz HJ, et al. CORDIAL: cognitive rehabilitation and cognitive-behavioral treatment for early dementia in Alzheimer disease: a multicenter, randomized, controlled trial. Alzheimer Dis Assoc Disord 2012;26:246-253.

5. World Health Organization. The ICD-10 Classification of Mental and Behavioural Disorders: Clinical Descriptions and Diagnostic Guidelines. Geneva: World Health Organization; 1992.

6. Stern C, Munn Z. Cognitive leisure activities and their role in preventing dementia: a systematic review. Int J Evid Based Healthc 2010;8:2-17.

7. Lee DY, Lee KU, Lee JH, Kim KW, Jhoo JH, Kim SY, et al. A normative study of the CERAD neuropsychological assessment battery in the Korean elderly. J Int Neuropsychol Soc 2004;10:72-81.

8. Lee JY, Lee DW, Cho SJ, Na DL, Jeon HJ, Kim SK, et al. Brief screening for mild cognitive impairment in elderly outpatient clinic: validation of the Korean version of the Montreal Cognitive Assessment. J Geriatr Psychiatry Neurol 2008;21:104-110.

9. Tappen RM, Rosselli M, Engstrom G. Evaluation of the Functional Activities Questionnaire (FAQ) in cognitive screening across four American ethnic groups. Clin Neuropsychol 2010;24:646-661.

10. Jhoo JH, Chi YK, Choi HJ, Han JW, Kim TH, Lee JJ, et al. A normative study of the disability assessment for dementia in community-dwelling elderly Koreans. Psychiatry Investig 2014;11:446-453.

11. Min SK, Kim KI, Lee CI, Jung YC, Suh SY, Kim DK. Development of the Korean versions of WHO Quality of Life scale and WHOQOLBREF. Qual Life Res 2002;11:593-600.

12. Lee DY, Lee KU, Lee JH, Kim KW, Jhoo JH, Youn JC, et al. A normative study of the mini-mental state examination in the Korean elderly. J Korean Neuropsychiatr Assoc 2002;41:508-525.

13. Aguirre E, Woods RT, Spector A, Orrell M. Cognitive stimulation for dementia: a systematic review of the evidence of effectiveness from randomised controlled trials. Ageing Res Rev 2013;12:253-262.

14. Bahar-Fuchs A, Clare L, Woods B. Cognitive training and cognitive rehabilitation for mild to moderate Alzheimer's disease and vascular dementia. Cochrane Database Syst Rev 2013;6:Cd003260.

15. Morris JC. The Clinical Dementia Rating (CDR): current Version and Scoring Rules. Neurology 1993;43:2412-2414.

16. Bahar-Fuchs A, Clare L, Woods B. Cognitive training and cognitive rehabilitation for persons with mild to moderate dementia of the Alzheimer's or vascular type: a review. Alzheimers Res Ther 2013;5:35.

17. Milders M, Bell S, Lorimer A, MacEwan T, McBain A. Cognitive stimulation by caregivers for people with dementia. Geriatr Nurs 2013;34: 267-273.

18. Onder G, Zanetti O, Giacobini E, Frisoni GB, Bartorelli L, Carbone G, et al. Reality orientation therapy combined with cholinesterase inhibitors in Alzheimer's disease: randomised controlled trial. Br J Psychiatry 2005;187:450-455.

19. Quayhagen MP, Quayhagen M, Corbeil RR, Hendrix RC, Jackson JE, Snyder L, et al. Coping with dementia: evaluation of four nonpharmacologic interventions. Int Psychogeriatr 2000;12:249-265. 